\title{
Laparoscopic left and right adrenalectomy from an anterior approach - is there any difference? Outcomes in 176 consecutive patients
}

\author{
Lukas Kokorak $^{1}$, Marek Soltes ${ }^{2}$, Peter Vladovic ${ }^{1}$, Lubomir Marko ${ }^{1}$ \\ ${ }^{1}$ Department of Miniinvasive Surgery and Endoscopy, F.D. Roosevelt Hospital, Banska Bystrica, Slovak Republic \\ ${ }^{2} 1^{\text {st }}$ Department of Surgery, University of Pavol Jozef Safarik, Kosice, Slovak Republic
}

Videosurgery Miniinv 2016; 11 (4): 268-273

DOI: https://doi.org/10.5114/wiitm.2016.64767

\begin{abstract}
Introduction: Traditionally, in open surgery, right adrenalectomy is considered technically more demanding than its left-sided counterpart. This belief is supposed to be attributable mainly to different anatomic characteristics of the adrenal veins. Whether this opinion is also correct for laparoscopic adrenalectomy remains elusive.

Aim: To compare the outcomes of left versus right laparoscopic adrenalectomy from an anterior approach.

Material and methods: Retrospective statistical analysis of a prospectively compiled database of consecutive patients undergoing laparoscopic adrenalectomy in a single center with focus on potential differences in the left-versus right-sided procedure in terms of demographic parameters, tumor size, operating time, occurrence of serious intraoperative complications, conversion, length of hospital stay and re-operation rate.

Results: One hundred seventy-six patients underwent elective laparoscopic adrenalectomy - 80 left-sided (45.45\%) and 96 right-sided (54.55\%). No significant difference was found between the groups in terms of age $(54.09 \pm 11.2$ vs. $56.27 \pm 11.6 ; p=0.2)$, tumor size ( $3.39 \pm 1.86$ vs. $3.26 \pm 1.66$; $p=0.64$ ), operating time ( $71.84 \pm 22.33$ vs. 72.06 $\pm 30.99 ; p=0.95)$, occurrence of serious intraoperative complications (7.5\% vs. $10.4 \% ; p=0.5)$, conversion $(1.25 \%$ vs. $1.04 \% ; p=0.9)$, length of hospital stay $(4.52 \pm 1.30$ vs. $4.37 \pm 1.91 ; p=0.55)$ or reoperation rate ( $5 \%$ vs. $1 \% ; p=$ $0.11)$. There was no mortality.

Conclusions: No significant difference was found between the left and right laparoscopic adrenalectomy in terms of operating time, occurrence of serious intraoperative complications, conversion rate or postoperative outcome. Therefore, the opinion that the right-sided procedure is more difficult does not seem to be justified for laparoscopic adrenalectomy from the anterior approach.
\end{abstract}

Key words: adrenalectomy, laparoscopic, right vs. left, complications, adrenal tumors.

\section{Introduction}

A minimally invasive approach towards such deep-seated organs as the adrenal glands represents a very appealing alternative for surgeons because no other surgical procedure than open adrenalectomy shows a greater difference between the small size of the organ to be removed and the extent of the skin incision needed. Therefore, since its introduction in 1992 [1], laparoscopic adrenalectomy has become more and more utilized, benefiting from a favorable postoperative course, decreased morbidity and better cosmetic result, thus progressively becoming the gold standard for the operation of adrenal glands [2]. 
Although in general, indications for laparoscopic adrenalectomy are no different from the open procedure, the size of the adrenal tumors still scheduled for laparoscopic approach remains somewhat controversial [3]. Despite several studies having shown that large tumors do not represent a contraindication for a laparoscopic procedure [4-7], efficacy has been clearly demonstrated for tumor sizes up to $12 \mathrm{~cm} \mathrm{[6].} \mathrm{The} \mathrm{role} \mathrm{of} \mathrm{laparoscopic} \mathrm{adrenalectomy}$ in the treatment of patients with adrenocortical carcinoma is another burning issue due to the doubts about the adequate oncological radicality of the surgical performance. However, data exist supporting the view that there is no significant difference in oncological outcome compared to open surgery [8].

It has been demonstrated that laparoscopic adrenalectomy for pheochromocytoma is more difficult compared to other adrenal tumors [9]. Traditionally, in open surgery, right adrenalectomy is considered technically more demanding than its left-sided counterpart. This belief is supposed to be attributable mainly to different anatomic characteristics of the adrenal veins, as the right adrenal gland vein is relatively short and drains directly into the vena cava inferior posterolaterally. Obviously, this may cause technical difficulties. Whether such an opinion is also correct for laparoscopic adrenalectomy remains elusive $[10,11]$.

\section{Aim}

The aim of the study is to compare left versus right laparoscopic adrenalectomy from the anterior approach and determine whether there are significant differences in terms of operating time, occurrence of serious intraoperative complications, conversion and postoperative outcome determined by the length of hospital stay and re-operation rate.

\section{Material and methods}

Consecutive patients undergoing elective laparoscopic adrenalectomy at the tertiary referral center from June 2006 until June 2016 were included in the study on the intention-to-treat basis, regardless of any other parameters, with no exclusions. Indications were justified by endocrinologists.

All the procedures were performed by two experienced laparoscopic surgeons in the standardized manner, with the anterior transperitoneal approach. The patient is positioned supine on the back, the operating surgeon stands between the legs of the patient (French position), the 4-port technique is used with an optional additional port if needed, and a $10 \mathrm{~mm} 30^{\circ}$ optic is used.

\section{Right-sided procedure}

The liver is elevated with the aid of the retractor, any adhesions of the gallbladder/omentum are divided, and the lateral hinge of the right liver lobe is aborted, in order to have a good high subhepatic access in the lateral area. After identification of the kidney, its upper edge is verified to define the space to look for the adrenal gland. Opening of the retroperitoneum along the edge of the liver follows, from the gallbladder towards the lateral abdominal wall. As soon as the tissue of the adrenal gland is identified, blunt dissection starts in the area of the lower pole along the medial surface superior towards the liver to identify the principal adrenal vein to be divided in between the clips. Caution is necessary as it drains directly into the vena cava inferior, its course is short and confluence often posterolateral, so as to avoid iatrogenic injury with profuse bleeding. After the vein is secured and divided, the gland is dissected completely with the aid of an ultrasonic scalpel to ensure perfect hemostasis. The specimen is then placed into the endobag and extracted from the abdominal cavity through the umbilical port.

\section{Left-sided procedure}

The splenic flexure of the colon is released, splenocolic and splenorenal ligaments divided, and the partially mobilized spleen elevated with the retractor orally. The left kidney is identified and the space above its upper pole verified to look for the adrenal gland. Caution is necessary not to misjudge it for the tail of the pancreas as the tissue characteristics are quite similar, sometimes leading to confusion, particularly in obese patients. Retraction and dissection of the pancreatic tail must be gentle in order to avoid postoperative pancreatitis/fistula due to contusion/ disruption. As soon as the tissue of the adrenal gland is identified, blunt dissection starts in the area of the lower pole along the medial surface heading superior to identify the principal adrenal vein to be divided in between the clips. Caution is necessary as it drains into the left renal vein and may be intimate to the tail of the pancreas and the splenic vasculature. The rest of the procedure is the same as during the right adrenalectomy described above. 
To compare left vs. right laparoscopic adrenalectomy from the anterior approach, data were extracted from the prospectively compiled institutional database including patient demographics (age, gender), site of surgery, tumor characteristics (size measured in centimeters based on the preoperative imaging, hormonal activity, histological finding), operating time (skin-to-skin in minutes), occurrence of serious intraoperative complications, conversion, length of hospital stay (days) and reoperation rate (including the reasons). All the parameters were recorded by a researcher independent from the operating surgeon.

\section{Statistical analysis}

For statistical analysis, obtained data were divided into two groups based on the site of surgery (left vs. right adrenalectomy). Defined data sets were then subsequently tested for significant differences with the level of statistical significance set at $95 \%$ $(p<0.05)$. Statistical tests used included the unpaired $t$-test and Mann-Whitney $U$ test for continuous and Fisher's exact test for binary variables.

\section{Results}

During the study period, 176 patients underwent laparoscopic adrenalectomy - 80 (45.45\%) left-sided and 96 (54.55\%) right-sided. Seventy one (40.34\%) patients were diagnosed with hormonally active tumors, associated with overproduction of aldosterone in 42 (59.1\%), overproduction of cortisol in 18 (25.4\%), pheochromocytoma in 8 (11.3\%), adrenogenital syndrome in $2(2.8 \%)$ and MEN Ila syndrome in 1 case (1.4\%), while 105 (59.66\%) lesions were hormonally inactive.

Average age of the patients was $55.33 \pm 11.42$ years (54.09 \pm 11.2 vs. $56.27 \pm 11.6 ; p=0.2), 110$ were women $(62.5 \%)$ and 66 men (37.5\%); no significant difference was noted in patient demographics between the left and right adrenalectomy groups. Overall the average tumor size recorded was 3.31 $\pm 1.74 \mathrm{~cm}$, without any statistical difference between the groups ( $3.39 \pm 1.86$ vs. $3.26 \pm 1.66$; $p=0.64$ ).

Average overall skin-to-skin operating time was $72.15 \pm 23.85 \mathrm{~min}(71.84 \pm 22.33$ vs. $72.06 \pm 30.99$; $p=0.95)$. Serious intraoperative complications occurred in 16 (9.09\%) cases, 6 (7.5\%) in the left ( $4 \times$ bleeding, $2 \times$ pancreatic injury) and 10 (10.4\%) in the right adrenalectomy ( $7 \times$ bleeding, $3 \times$ liver injury)
- the difference did not reach statistical significance $(p=0.5)$. Conversion occurred in $2(1.14 \%)$ patients - one in the left (severe laceration of the pancreatic tail) and one in the right-sided procedure (extensive adhesions in the abdominal cavity after previous procedures) ( $p=0.9$ ).

Histological evaluation verified adrenocortical adenoma in 129 (73.3\%), hyperplasia in 14 (7.96\%), pheochromocytoma in 12 (6.82\%), myelolipoma in 8 (4.55), cysts in 5 (2.85\%), ganglioneuroma in $3(1.7 \%)$, metastatic involvement in $3(1.7 \%)$, neurofibroma in 1 (0.57\%) and hematoma in 1 (0.57\%) case.

Average overall length of hospital stay was 4.43 \pm 1.66 days $(4.52 \pm 1.30$ vs. $4.37 \pm 1.91 ; p=0.55)$. Re-operation was required in 5 (2.84\%) patients, in all cases due to hemoperitoneum, 4 patients in the left and one in the right adrenalectomy group (5\% vs. $1 \% ; p=0.11$ ). There was no mortality.

Statistical evaluation of the differences in age, operating time, size of the tumor and length of hospital stay between the groups is summarized in Table I.

\section{Discussion}

Minimally invasive surgical procedures such as laparoscopic adrenalectomy tend to reduce operative trauma, thus resulting in lower blood loss, better convalescence, lower analgesics consumption, decreased wound-related morbidity, shorter hospital stay and a better cosmetic outcome compared to open surgery [12]. Yet another advantage of the laparoscopic approach to the adrenal glands appears to be in an improved magnified view allowing for precise, hemostatic dissection in a minimally invasive fashion [13, 14].

Various minimally invasive techniques have been reported for adrenalectomy. The retroperitoneoscopic approach seems optimal in patients with small adrenal tumors [15]. Myśliwiec et al. recommend retroperitoneoscopic adrenalectomy for benign adrenal tumors $<6 \mathrm{~cm}$ in particular and highlight the possibility of simultaneous bilateral adrenalectomy if a posterior retroperitoneoscopic approach is used [16]. Some reports describe laparoendoscopic single site (LESS) adrenalectomy as more time consuming compared to the standard laparoscopic procedure $[17,18]$. The LESS adrenalectomy seems feasible in selected cases, especially small left-sided tumors in 
Table I. Left versus right adrenalectomy

\begin{tabular}{|c|c|c|c|c|c|c|c|c|c|c|c|c|}
\hline \multirow[t]{2}{*}{ Parameter } & \multicolumn{3}{|c|}{ Age } & \multicolumn{3}{|c|}{ Operating time [min] } & \multicolumn{3}{|c|}{ Size of tumor $[\mathrm{cm}]$} & \multicolumn{3}{|c|}{$\begin{array}{l}\text { Length of hospital stay } \\
\text { [days] }\end{array}$} \\
\hline & Total & Left & Right & Total & Left & Right & Total & Left & Right & Total & Left & Right \\
\hline Average & 55.33 & 54.09 & 56.27 & 72.15 & 71.84 & 72.06 & 3.31 & 3.39 & 3.26 & 4.43 & 4.52 & 4.37 \\
\hline Standard deviation & 11.42 & 11.20 & 11.60 & 27.41 & 22.33 & 30.99 & 1.74 & 1.86 & 1.66 & 1.66 & 1.30 & 1.91 \\
\hline Minimum & 18 & 29 & 18 & 30 & 40 & 30 & 1 & 1 & 1 & 3 & 3 & 3 \\
\hline Maximum & 80 & 77 & 80 & 180 & 150 & 180 & 12 & 12 & 8 & 18 & 18 & 11 \\
\hline Median & 56 & 55 & 58 & 70 & 70 & 65 & 3 & 3 & 3 & 4 & 4 & 4 \\
\hline Mode & 53 & 43 & 60 & 75 & 75 & 75 & 3 & 3 & 3 & 4 & 4 & 3 \\
\hline Significance & - & \multicolumn{2}{|c|}{$p=0.20$} & - & \multicolumn{2}{|c|}{$p=0.95$} & - & \multicolumn{2}{|c|}{$p=0.64$} & - & \multicolumn{2}{|c|}{$p=0.55$} \\
\hline
\end{tabular}

thin patients with no history of previous abdominal operations, but may require an additional port in half of the cases [19]. Robot-assisted adrenalectomy is described to have a shorter hospital stay, less blood loss, and lower occurrence of postoperative complications [20, 21].

Laparoscopic adrenalectomy may be performed from either a lateral (patient in lateral decubitus) or anterior (patient supine on the back) approach, the latter being used less frequently [22]. Although the anterior approach is advocated by fewer centers, the experience of our institution with this technique is good, and it is applied as a method of choice for all the patients scheduled for elective adrenalectomy in whom the indication is justified.

As for the indications, hormonally active tumors are indicated for surgery by the endocrinologist. For hormonally inactive lesions (incidentalomas), a generally accepted recommendation is to operate on those larger than $6 \mathrm{~cm}$. Lesions smaller than $4 \mathrm{~cm}$ and defined as low risk by imaging criteria are unlikely to have malignant potential and should not generally be resected. The need and strategy for routine follow-up in this group remain not clearly defined. For lesions between $4 \mathrm{~cm}$ and $6 \mathrm{~cm}$, either close follow-up or adrenalectomy is considered a reasonable approach. Surgery should be strongly considered if the imaging findings, including rapid growth rate and/or decreased lipid content, are confirmed [23]. Considering laparoscopic adrenalectomy a standard procedure replacing open surgery for benign lesions, Castillo et al. advocate it for tumors sized equal to or less than $12 \mathrm{~cm}$ [24]. This observation is also valid in our series with lesions sized from 1 to $12 \mathrm{~cm}$.
Although the indications for supposedly malignant tumors are still controversial, Pujol et al. described their limited experience as early as in 1999 [25]. Beisa et al. demonstrated recently the feasibility of two-stage laparoscopic adrenalectomy for bilateral pheochromocytoma, with one of the tumors larger than $12 \mathrm{~cm}$ without signs of invasion to adjacent structures and organs [26].

Because of the anatomic differences between the right and left adrenal glands, most notably the different venous drainage patterns, surgical approaches are somewhat different for right and left adrenalectomy. Right-sided adrenalectomy is generally believed to be more difficult because of the proximity of dissection to the inferior vena cava and duodenum and the short adrenal vein. Whether such an assumption is true in laparoscopic adrenalectomy, however, remains elusive. Rieder et al. retrospectively reviewed 163 laparoscopic adrenalectomies, 109 on the left and 54 on the right side. The average operating time for left-sided procedure was 187 min (62-475) - significantly longer (by $31 \mathrm{~min}$ ) compared to $156 \min (60-365)$ on the right side. No difference was detected in complication and conversion rates [10]. Lezoche et al. also observed shorter operating times for right adrenalectomy (80 vs. $109 \mathrm{~min})$, although the difference was not statistically significant [27]. Similar results are reported in several others studies, where the mean operating time for right adrenalectomy is shorter compared to the left-sided procedure [28-30]. Our results also confirm that there is at least no statistical difference between the length of operating time for left vs. right laparoscopic adrenalectomy. The described 
observations may probably be explained by the need of colonic splenic flexure mobilization during laparoscopic left adrenalectomy, which seems to be more complex compared to open surgery, as well as by the potential problems of proper identification of the left adrenal gland in obese patients and it possibly being mistaken for the pancreatic tail in some cases.

Although laparoscopic adrenalectomy is considered an advanced procedure, conversion is rarely required in our experience (1.14\%). Paganini et al. reported a conversion rate of $3.22 \%$ due to extensive adhesions and hemorrhage, and Pillinger et al. experienced the necessity to convert in $5.1 \%$ of cases $[28,29]$. Similar results were observed by Henry et al., describing a $5 \%$ conversion rate due to intraoperatively diagnosed malignancy, inability to proceed because of difficult dissection and iatrogenic pneumothorax [30].

As for the incidence of serious intraoperative and postoperative complications and reoperation rates, the literature data are quite heterogeneous. Based on the conclusions of two meta-analyses it seems justified that laparoscopic adrenalectomy is associated with relatively low rates of perioperative complications [31, 32]. Henry et al. report $7.5 \%$ significant complications including intraabdominal hemorrhage requiring laparotomy and/or transfusion therapy, abdominal wall hematoma, major intraoperative hemorrhage, partial infarction of the spleen, iatrogenic pneumothorax, capsular rupture of the tumor and major deep venous thrombosis [30]. The most serious and feared intraoperative complication of laparoscopic right adrenalectomy is hemorrhage from the adrenal vein, inferior vena cava or gland itself, which can cause severe blood loss and may require conversion to an open procedure. Several studies consider early clipping of the main adrenal vein along with avoiding any manipulation of the gland before this is achieved as the principal technical advice $[33,34]$. For laparoscopic left adrenalectomy, injuries to the pancreatic tail and hemorrhage from either the adrenal vein or the splenic vasculature are the principal intraoperative challenges. Varkarakis et al. reported as many as $8 \%$ of distal pancreatic injuries with laparoscopic adrenalectomy [35]. Our observation yields better results, with $1.13 \%$ overall and $2.5 \%$ specifically for left-sided procedures. No statistically significant difference was found in our series in terms of incidence of serious intraoperative complications and reoperation rates between left and right laparoscopic adrenalectomy, despite there being more intraoperative complications in the right and more reoperations in the left-sided procedure.

When analyzing the data from the large case series and meta-analyses $[8,20,30-32]$ it can be concluded that our overall results seem to confirm the standardized surgical technique. Therefore, the results of the study should not be biased by either an individual or institutional learning curve. Also of note, there was no statistical difference in age of the operated patients or tumor size, so these potential confounding parameters may be excluded. All consecutive patients undergoing laparoscopic adrenalectomy were included in the analysis, which was carried out on an intention-to-treat basis, so selection bias is limited. The main drawbacks of the study are the retrospective design of the analysis (despite the database being compiled in a prospective manner) and limited external validity due to the unicentric character of the study performed. Prospective multicentre trials might yield more robust evidence in future.

\section{Conclusions}

No significant difference was found between the left and right laparoscopic adrenalectomy in terms of operating time, occurrence of serious intraoperative complications, conversion rate or postoperative outcome. Therefore, the opinion that the right-sided procedure is more difficult does not seem to be justified for laparoscopic adrenalectomy from an anterior approach. The technique is well established, reliable and safe provided there is no negative influence of either an individual or institutional learning curve.

\section{Conflict of interest}

The authors declare no conflict of interest.

\section{References}

1. Gagner M, Lacroix AB. Laparoscopic adrenalectomy in Cushing's syndrome and pheochromocytoma. N Engl J Med 1992; 327: 1033-7.

2. Smith CD, Weber CJ, Amerson JR. Laparoscopic adrenalectomy: new gold standard. World J Surg 1999; 23: 389-96.

3. Aksakal N, Agcaoglu O, Barbaros U, et al. Safety and feasibility of laparoscopic adrenalectomy: what is the role of tumour size? A single institution experience. J Minim Access Surg 2015; 11: 184-6.

4. Henry JF, Defechereux T, Gramatica L, et al. Should laparoscopic approach be proposed for large and/or potentially malignant adrenal tumors? Langenbecks Arch Surg 1999; 384: 366-9. 
5. Hobart MG, Gill IS, Schweizer D, et al. Laparoscopic adrenalectomy for large-volume (> or $=5 \mathrm{~cm}$ ) adrenal masses. J Endourol 2000; 14: 149-54.

6. MacGillivray DC, Whalen GF, Malchoff CD, et al. Laparoscopic resection of large adrenal tumors. Ann Surg Oncol 2002; 9: 480-5.

7. Al-Zahrani HM. Laparoscopic adrenalectomy: an update. Arab J Urol 2012; 10: 56-65.

8. Brix D, Allolio B, Fenske W, et al. Laparoscopic versus open adrenalectomy for adrenocortical carcinoma: surgical and oncolog. ic outcome in 152 patients. Eur Urol 2010; 58: 609-15.

9. Natkaniec M, Pędziwiatr M, Wierdak M, et al. Laparoscopic adrenalectomy for pheochromocytoma is more difficult compared to other adrenal tumor. Videosurgery Miniinv 2015; 10: 466-71.

10. Rieder JM, Nisbet AA, Wuerstle MC, et al. Differences in left and right laparoscopic adrenalectomy. JSLS 2010; 14: 369-73.

11. Cianci P, Fersini A, Tartaglia N, et al. Are there differences between the right and left laparoscopic adrenalectomy? Our ex perience. Ann Ital Chir 2016; 87: 242-6.

12. Bolli M, Oertli D, Staub JJ, et al. Laparoscopic adrenalectomy: the new standard? Swiss Med Wkly 2002; 132: 12-6.

13. Lev-Chelouche D, Sagie B, Keidar A, et al. Laparoscopic adrenalectomy: indications, technique, complications and follow-up. Isr Med Assoc J 2003; 5: 101-4.

14. Raeburn CD, McIntyre RC. Laparoscopic approach to adrenal and endocrine pancreatic tumors. Surg Clin North Am 2000 80: $1427-41$.

15. Bonjer HJ, Lance JF, Kazemier G, et al. Comparison of three techniques for adrenalectomy. Br J Surg 1997; 84: 679-82.

16. Myśliwiec P, Safiejko M, Łukaszewicz J, et al. Videoscopic adrenalectomy - when does retroperitoneal seem better? Videosurgery Miniinv 2014; 9: 226-33.

17. Rane A, Cindolo L, Schips L, et al. Laparoendoscopic single site (LESS) adrenalectomy: technique and outcomes. World J Urol 2012; 30: 597-604

18. Yang W, Yao H, Bin-Shen L, et al. Laparoendoscopic single-site retroperitoneoscopic adrenalectomy versus conventional retroperitoneoscopic adrenalectomy in obese patients. J Endourol 2016; 30: 306-11.

19. Hora T, Ürge T, Stránský P, et al. Laparoendoscopic single-site surgery adrenalectomy - own experience and matched casecontrol study with standard laparoscopic adrenalectomy. Videosurgery Miniinv 2014; 9: 596-602.

20. Brandao LF, Autorino R, Laydner H, et al. Robotic versus laparoscopic adrenalectomy: a systematic review and meta-analysis. Eur Urol 2014; 65: 1154-61.

21. Taskin HE, Berber E. Robotic adrenalectomy. J Surg Oncol 2013; 19: 162-6.

22. Scoglio D, Balla A, Paci M, et al. Laparoscopic transperitoneal anterior adrenalectomy. Ann Ital Chir 2013; 84: 411-6.

23. Grumbach MM, Biller BM, Braunstein GD, et al. Management of the clinically inapparent adrenal mass ("Incidentaloma"). Ann Intern Med 2003; 138: 424-9.

24. Castillo O, Sánchez-Salas R, Vidal I. Laparoscopic adrenalectomy. J Endocrinol Invest 2009; 32: 57-62.

25. Pujol J, Viladrich M, Rafecas A, et al. Laparoscopic adrenalectomy: a review of 30 initial cases. Surg Endosc 1999; 13: 488-92.
26. Beisa V, Klimovskij M, Simutis G, et al. Two-stage bilateral laparoscopic adrenalectomy for large pheochromocytomas. Videosurgery Miniinv 2014; 9: 110-4.

27. Lezoche E, Guerrieri M, Crosta F, et al. Perioperative results of 214 laparoscopic adrenalectomies by anterior transperitoneal approach. Surg Endosc 2008; 22: 522-6.

28. Paganini AM, Balla A, Guerrieri M, et al. Laparoscopic transperitoneal anterior adrenalectomy in pheochromocytoma: experience in 62 patients. Surg Endosc 2014; 28: 2683-9.

29. Pillinger SH, Christopher PB, Sidhu S. Laparoscopic adrenalectomy: a 6-year experience of 59 cases. ANZ J Surg 2002; 72: 467-70.

30. Henry JF, Defechereux T, Raffaelli M, et al. Complications of laparoscopic adrenalectomy: results of 169 consecutive procedures. World J Surg 2000; 24: 1342-6.

31. Nigri G, Rosman AS, Petrucciani N, et al. Meta-analysis of trials comparing laparoscopic transperitoneal and retroperitoneal adrenalectomy. Surgery 2013; 153: 111-9.

32. Tang K, Li H, Xia D, et al. Robot-assisted versus laparoscopic adrenalectomy: a systematic review and meta-analysis. I Laparoendosc Adv Surg Tech 2015; 25: 187-95.

33. Lezoche E, Guerrieri M, Feliciotti F, et al. Anterior, lateral and posterior retroperitoneal approaches in endoscopic adrenalectomy. Surg Endosc 2002; 16: 96-9.

34. Janatschek G, Neumann HP. Laparoscopic surgery for pheochromocytoma. Urol Clin North Ann 2001; 28: 97-105.

35. Varkaris IM, Allaf ME, Bhayani SB, et al. Pancreatic injuries during laparoscopic urologic surgery. Urology 2004; 64: 1089-93.

Received: 8.12.2016, accepted: 10.12.2016. 